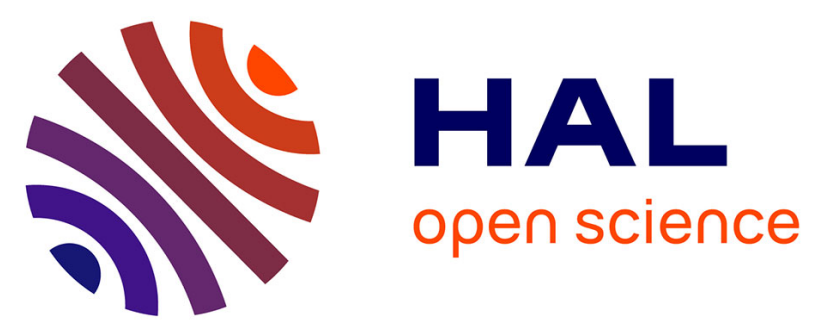

\title{
Uncovering the benefits of fluctuating thermal regimes on cold tolerance of drosophila flies by combined metabolomic and lipidomic approach
}

Hervé Colinet, D Renault, Marion Javal, Petra Berková, Vladimir Koštál

\section{- To cite this version:}

Hervé Colinet, D Renault, Marion Javal, Petra Berková, Vladimir Koštál. Uncovering the benefits of fluctuating thermal regimes on cold tolerance of drosophila flies by combined metabolomic and lipidomic approach. Biochimica et Biophysica Acta Molecular and Cell Biology of Lipids, 2016, 1861 (11), pp.1736-1745. 10.1016/j.bbalip.2016.08.008 • hal-01371831

\section{HAL Id: hal-01371831}

\section{https://hal-univ-rennes1.archives-ouvertes.fr/hal-01371831}

Submitted on 27 May 2020

HAL is a multi-disciplinary open access archive for the deposit and dissemination of scientific research documents, whether they are published or not. The documents may come from teaching and research institutions in France or abroad, or from public or private research centers.
L'archive ouverte pluridisciplinaire HAL, est destinée au dépôt et à la diffusion de documents scientifiques de niveau recherche, publiés ou non, émanant des établissements d'enseignement et de recherche français ou étrangers, des laboratoires publics ou privés. 


\title{
Uncovering the benefits of fluctuating thermal regimes on cold tolerance of drosophila flies by combined metabolomic and lipidomic approach
}

\author{
Hervé Colinet $^{\mathrm{a}, *}$, David Renault ${ }^{\mathrm{a}}$, Marion Javal ${ }^{\mathrm{b}}$, Petra Berková ${ }^{\mathrm{c}}$, Petr Šimek ${ }^{\mathrm{c}}$, Vladimír Koštál ${ }^{\mathrm{c}}$ \\ a Université de Rennes 1, UMR CNRS 6553 ECOBIO, 263 avenue du Général-Leclerc, 35042, Rennes, France \\ b URZF, INRA, 45075, Orléans, France \\ ' Institute of Entomology, Biology Centre of the Czech Academy of Sciences, Branišovská 31, 370 05, České Budějovice, Czech Republic
}

\section{A R T I C L E I N F O}

\section{Article history:}

Received 4 May 2016

Received in revised form 21 July 2016

Accepted 15 August 2016

Available online 16 August 2016

\section{Keywords:}

Cold stress

fluctuating thermal regimes

recovery

Omics

Drosophila

\begin{abstract}
A B S T R A C T
When exposed to constant low temperatures (CLTs), insects often suffer from cumulative physiological injuries that can severely compromise their fitness and survival. Yet, mortality can be considerably lowered when the cold stress period is interrupted by periodic warm interruption(s), referred to as fluctuating thermal regimes, FTRs. In this study, we have shown that FTRs strongly promoted cold tolerance of Drosophila melanogaster adults. We then assessed whether this marked phenotypic shift was associated with detectable physiological changes, such as synthesis of cryoprotectants and/or membrane remodeling. To test these hypotheses, we conducted two different time-series Omics analyzes in adult flies submitted to CLTs vs. FTRs: metabolomics (GC/MS) and lipidomics (LC/ESI/MS) targeting membrane phospholipids. We observed increasing levels in several polyhydric alcohols (arabitol, erythritol, sorbitol, mannitol, glycerol), sugars (fructose, mannose) and amino acids (serine, alanine, glutamine) in flies under CLT. Prolonged exposure to low temperature was also associated with a marked deviation of metabolic homeostasis and warm interruptions as short as $2 \mathrm{~h}$ were sufficient to periodically return the metabolic system to functionality. Lipidomics revealed an increased relative proportion of phosphatidylethanolamines and a shortening of fatty acyl chains in flies exposed to cold, likely to compensate for the ordering effect of low temperature on membranes. We found a remarkable correspondence in the time-course of changes between the metabolic and phospholipids networks, both suggesting a fast homeostatic regeneration during warm intervals under FTRs. In consequence, we suggest that periodic opportunities to restore system-wide homeostasis contribute to promote cold tolerance under FTRs.
\end{abstract}

(c) 2016 Elsevier B.V. All rights reserved.

\section{Introduction}

Temperature affects virtually all aspects of ectotherms' life, including behavior, physiological processes, metabolic and signaling pathways and gene transcription [1]. For small ectotherms like insects, temperature can often reach stressing limits. Insects exposed to even relatively mild (sublethal) chilling for prolonged time may suffer from cumulative (so called indirect) physiological damages that can severely

Abbreviations: ANOVA, analysis of variance; CL, cardiolipin; CLT, constant low temperature; CO, constant control; FA, fatty acid; FTR, fluctuating thermal regime; GC/ MS, gas chromatography-mass spectrometry; LC/ESI/MS, liquid chromatography combined with electrospray ionization mass spectrometry; phospholipids, PLs; PC, phosphatidylcholine; PE, phosphatidylethanolamine; PG, phosphatidylglycerol; PI, phosphatidylinositol; PS, phosphatidylserine; LPC, lyso-PC; LPE, lyso-PE; LPG, lyso-PG; LPI, lyso-PI; MGDG, monogalactosyl diglyceride; PCA, principal component analysis; PC1, PC2 and PC3, first, second and third principal components; SFA, MUFA and PUFA, saturated, monounsaturated and polyunsaturated FA respectively; PUFA/MUFA, polyunsaturation ratio; UFA/SFA, unsaturation ratio; UI, unsaturation index.

* Corresponding author at: UMR CNRS 6553 Bât 14A, Université de Rennes1, 263 Avenue du Général Leclerc CS 74205, 35042, Rennes, France.

E-mail address: herve.colinet@univ-rennes1.fr (H. Colinet). compromise their fitness and survival. Studies of cold tolerance have generally been conducted under constant low temperatures (CLTs). However, the natural thermal environment is hardly ever stable, but rather fluctuates on scales ranging from hours to seasonal cycles. Not surprisingly, the responses of insects to constant temperatures significantly differ from those to fluctuating temperatures, and there is a growing body of literature describing the effects of thermal fluctuations on insect growth, life-history traits, stress tolerance and general physiology reviewed in [2].

Applying fluctuating temperatures during cold exposure can strongly affect insect's cold tolerance. Indeed, mortality is considerably lowered when a cold period is interrupted by brief warm interruption(s) (referred to as fluctuating thermal regime, FTR) [3-7]. Beneficial effects of FTRs have been reported when warm interruptions were applied on a daily basis [3-7] or just once within a prolonged cold stress $[8,9]$. Even short-term warm episodes (a few minutes to hours) are enough to mitigate cold-induced mortality [3,10-12]. The growing list of experimental evidence for the positive effect of FTRs suggests that mechanisms behind this process are highly conserved across insect taxa [2]. However, the benefits of FTRs hold true only for freeze- 
avoiding and chill-susceptible species. Indeed, periodic warming insects that survive in frozen state (freeze tolerant species) is detrimental because of repeated freeze-thaw cycles [2].

The physiological perturbations related to chilling are not yet fully characterized reviewed in [13]. Membranes are considered among the most thermally-sensitive macromolecular structures and are thus a primary target of chilling injuries. Chilling induces fluid to gel phase transition in cell membranes that can result in separation of phospholipids bilayer, change in permeability and sharp decline in the activity of membrane-bound enzymes [14-16]. These alterations can in turn severely compromise ion and water homeostasis across membranes [17-20], which causes neuromuscular alterations, loss of function, chill-coma, and ultimately chill injury and death [21-24]. Consequently, the remodeling of membrane lipids is a prime candidate mechanism underlying the positive effect of warm interruptions on insect cold tolerance under FTRs. Ectotherms typically compensate for temperatureinduced changes in viscosity of membrane lipids by homeoviscous adaptation [14,25], which involves remodeling the chemical composition of phospholipids (PLs). When compensation is not possible or feasible, the membrane remodeling aims at preventing occurrence of unregulated membrane phase transitions and, this way, preserving the structural integrity of membranes exposed to thermal stresses [15]. Whether membrane composition is adaptable under the rapidly changing thermal conditions that characterize FTRs is not known. Another potential mechanism occurring during FTRs is the mobilization of cryoprotectants. These compatible solutes are known to protect against chilling injuries [23]. Cryoprotectants include polyhydric alcohols, sugars, and some free amino acids. A primary function of these molecules relates to their colligative effect at high concentrations, but they also play non-colligative protective roles at low concentrations by stabilizing macromolecules and membranes [26-29]. Therefore, we decided to directly test whether cryoprotectants can be mobilized during cold period and warm interruptions under FTRs.

Although the mechanisms underlying the benefits of FTRs are not yet fully known, a generally accepted assumption is that insects profit from periodic warming opportunities to physiologically recover from chilling injuries that accumulated during the preceding cold exposure $[2,30]$. However, direct evidence of such a physiological repair is still missing. Environmental stresses transiently disturb cellular homeostasis, and in response, cells activate a complex response to progressively restore homeostasis [31]. Time-series metabolomics can be useful to monitor metabolic homeostasis status (metabolic trajectories) during stress or recovery [3234]. By analogy, analysis of lipidomic trajectories derived from large-scale temporal profiling of PLs can be used to track the dynamics of the homeostatic response in membranes during stress and recovery.

In this study, we applied CLTs and FTRs to adult Drosophila melanogaster flies and observed strong reduction of the cold-induced mortality under FTRs. Next, we hypothesized that this phenotypic change would be associated with detectable physiological signatures. Several hypotheses were tested: first, we expected adjustments of the concentrations of molecules with potential cryoprotective functions. Second, we expected membrane restructuring during either cold period or warm intervals in a direction that would support an increased cold tolerance. Finally, we assumed that FTRs will allow a fast homeostatic regeneration process. Specifically, we hypothesized that (i) metabolic and lipidomic trajectories will deviate markedly from the control state during cold periods and (ii) warm intervals will permit a return towards the initial homeostatic state.

\section{Material and methods}

\subsection{Fly culture}

We conducted the experiments on a mass-bred $D$. melanogaster line derived from two wild populations collected in October 2010 in Brittany (France). Flies were maintained in laboratory in $200 \mathrm{~mL}$ bottles at $25 \pm$
$1{ }^{\circ} \mathrm{C}$ (12 L:12D) on standard fly medium consisting of brewer yeast ( $80 \mathrm{~g} / \mathrm{L})$, sucrose $(50 \mathrm{~g} / \mathrm{L})$, agar $(15 \mathrm{~g} / \mathrm{L})$, and Nipagin ${ }^{\circledR}(8 \mathrm{~mL} / \mathrm{L})$. To generate flies for the experiments, groups of 15 mated females were allowed to lay eggs in $200 \mathrm{~mL}$ rearing bottles during a restricted period of $6 \mathrm{~h}$ under laboratory conditions. This controlled procedure allowed larvae to develop under uncrowded conditions. At emergence, adults were sexed visually (with an aspirator) without $\mathrm{CO}_{2}$ to avoid stress due to anesthesia [35]. Individuals used in all experiments were synchronized at the age of 6-d-old to avoid effects on stress tolerance traits in maturating young adults (<3-d-old) [36].

\subsection{Cold-survival}

Cold survival of adult flies was tested either under constant low temperatures (CLTs) or fluctuating thermal regimes (FTRs). Four different temperatures were used: $2,3,4$, and $5{ }^{\circ} \mathrm{C}$. Preliminary experiments revealed that flies fall into chill-coma at all of these temperatures. For FTRs, the temperatures were the same except that the cold period was interrupted daily by a short episode at $20^{\circ} \mathrm{C}$ for $2 \mathrm{~h}$. These short warm intervals are known to promote cold survival in other species reviewed in [2]. For each experimental condition, several groups of 15 flies were placed into vials that contained only agar. Pure agar diet was used in order to avoid any confounding effect due to re-nutrition of flies during warming intervals (possible under FTR only). For each experimental condition, ten vials of 15 flies were placed inside programmed thermo-regulated incubators (Model SANYO MIR-153) set at the requested temperature $\left(2,3,4\right.$, and $\left.5{ }^{\circ} \mathrm{C}\right)$ and thermal regime (CLT or FTR) (i.e. 150 flies per experimental condition). Temperature was checked using automatic recorders (Hobo ${ }^{\circledR}$ data logger, model U12012 , Onset Computer Corporation, accuracy $\pm 0.35^{\circ} \mathrm{C}$ ). The experiment lasted for 10 consecutive days ( $240 \mathrm{~h}$ ). Every $24 \mathrm{~h}$, a vial with 15 flies was removed from the cold-incubator and transferred to the laboratory conditions to score the survival after $4 \mathrm{~h}$ and also after $24 \mathrm{~h}$ of recovery post stress. Survival was scored as the number of flies that could stand on legs. All these experiments were performed with males and females separately. The experiment was performed in 2013 and a complete replication was performed in 2014

\subsection{Experimental design for Omics profiling}

Because the survival assays revealed that benefits of FTRs were particularly manifested in females exposed to $5{ }^{\circ} \mathrm{C}$, we decided to use only females in this thermal condition for monitoring metabolic and lipidomic profiles in specific follow-up experiment (see Fig. 1). Only the first three days of exposure were targeted for Omics profiling to ensure the complete absence of dead individuals in samples. Females were exposed to cold treatments (CLT and FTR), and a third group of females was submitted to constant $20{ }^{\circ} \mathrm{C}$ as control (CO). Females were all virgin, synchronized at the age of 6-d-old and placed in incubators in vials with only agar. In CLT treatment, flies were assessed after 1, 2, and 3 days of cold stress at $5{ }^{\circ} \mathrm{C}$ (codes: CLT1 to 3) (see Fig. 1). The FTR treatment was divided into two sub-treatments: i) flies monitored at the end of the cold period, just before the onset of the warm interval (codes: Fb1 to 3 for FTR "before") and ii) flies monitored after the recovery, at the end of the $2 \mathrm{~h}$ warm interval (codes: Fa 1 to 3 for FTR "after") (see Fig. 1). Finally, CO flies were monitored for 3 consecutive days at $20{ }^{\circ} \mathrm{C}$ (codes: $\mathrm{CO} 1$ to 3 ). For each time point, 14 replicates of 10 pooled females were used: 6 for metabolomics, 6 for lipidomics and 2 for checking the survival after 24 h recovery. Flies destined to biochemical analyzes were snap frozen in liquid $\mathrm{N}_{2}$ before being stored at $-80^{\circ} \mathrm{C}$ for extractions.

\subsection{Extractions and GC/MS-based metabolic profiling}

Each sample was weighed (Mettler Toledo UMX2, accurate to $1 \mu \mathrm{g}$ ) before metabolite extractions. Sample preparation and derivatization 


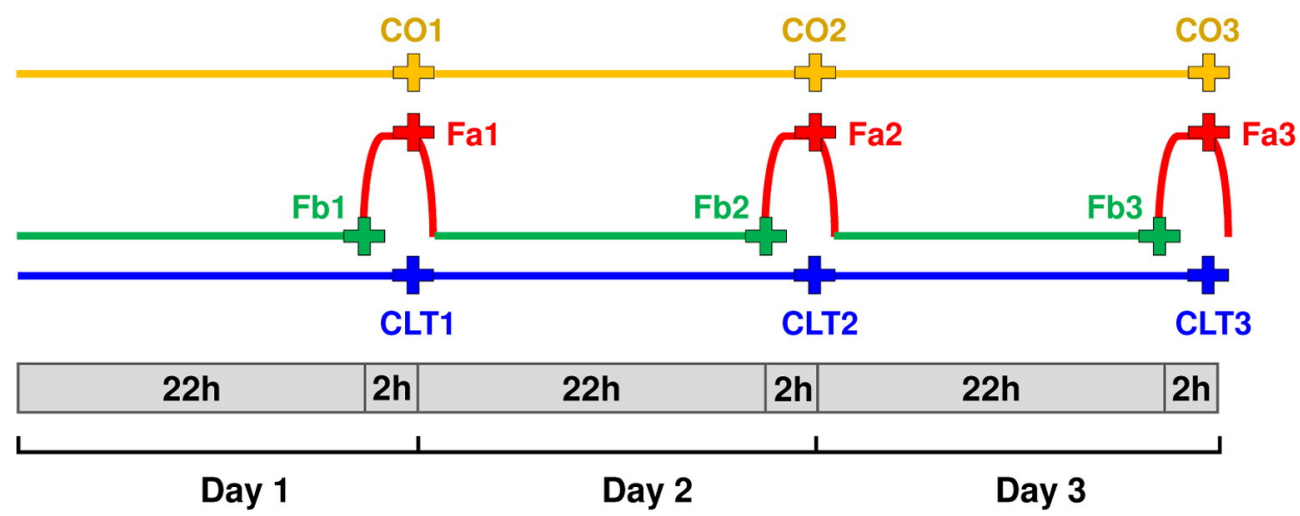

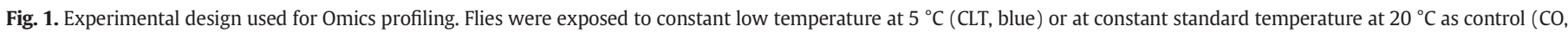

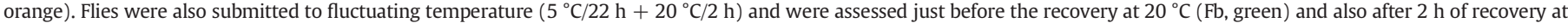
$20^{\circ} \mathrm{C}$ (Fa, red). Samples were taken for 3 consecutive days.

were performed as previously described $[33,37]$, with minor modifications. Briefly, after homogenization in $600 \mu \mathrm{L}$ of ice-cold methanolchloroform solution (2:1, v:v) and phase separation with $400 \mu \mathrm{L}$ of ultrapure water, a $100 \mu \mathrm{L}$ aliquot of the upper phase, which contained polar metabolites, was vacuum-dried. The dry residue was resuspended in $30 \mu \mathrm{L}$ of $20 \mathrm{mg} \mathrm{mL}^{-1}$ methoxyamine hydrochloride in pyridine before incubation under automatic orbital shaking at $40{ }^{\circ} \mathrm{C}$ for $60 \mathrm{~min}$. Then, $30 \mu \mathrm{L}$ of BSTFA were added and the derivatization was conducted at $40{ }^{\circ} \mathrm{C}$ for $60 \mathrm{~min}$ under agitation. A CTC CombiPal autosampler (GERSTEL GmbH and Co.KG, Mülheim an der Ruhr, Germany) was used, ensuring standardized sample preparation and timing. Metabolites were separated, identified and quantified using a GC/MS platform consisting of a Trace GC Ultra chromatograph and a Trace DSQII quadrupole mass spectrometer (Thermo Fischer Scientific Inc., Waltham, MA, USA). The oven temperature ranged from 70 to $170{ }^{\circ} \mathrm{C}$ at $5{ }^{\circ} \mathrm{C} \mathrm{min}-1$, from 170 to $280{ }^{\circ} \mathrm{C}$ at $7{ }^{\circ} \mathrm{C} \mathrm{min}{ }^{-1}$, from 280 to $320^{\circ} \mathrm{C}$ at $15^{\circ} \mathrm{C} \mathrm{min}^{-1}$, and then, the oven remained at $320^{\circ} \mathrm{C}$ for $4 \mathrm{~min}$. We completely randomized the injection order of the samples. All samples were run under the SIM mode rather than the full-scan mode. We therefore only screened for the 63 pure reference compounds included in our custom spectral database. Calibration curves for 63 pure reference compounds at $1,2,5,10,20,50,100,200,500,750,1000$, and $1500 \mu \mathrm{M}$ concentrations were run concurrently. Chromatograms were deconvoluted using XCalibur 2.0.7, and metabolite levels were quantified using the quadratic calibration curve for each reference compound and concentration. Quality controls at concentrations of $200 \mu \mathrm{M}$ were run every 15 samples. Arabinose (at $2 \mathrm{mM}$ ) was used as internal standard to account for potential loss during sample preparation. Calculated concentrations were adjusted according to internal standard.

\subsection{Extraction and LC/ESI/MS analysis of phospholipid composition}

Total lipids were extracted twice in $400 \mu \mathrm{L}$ of chloroform:methanol solution (2:1, v:v) and evaporated to dryness. Total lipid extract was then separated into polar and non-polar classes by dissolution and liquid-liquid extraction between $2 \mathrm{~mL}$ acetonitrile in water (80:20) and $2 \mathrm{~mL}$ hexane. The lower aqueous acetonitrile phase containing polar lipids was used as a source of phospholipids. The samples were analyzed using high performance liquid chromatography (LC) combined with electrospray ionization mass spectrometry (ESI/MS) as described in previous studies [38,39]. Briefly, a LTQ-XL mass spectrometer (Thermo Fisher Scientific) equipped with ESI, Accela 600 pump HPLC system, and Accela AS autosampler (Thermo Fisher Scientific, San Jose, CA, USA) was used. A volume of $200 \mu \mathrm{L}$ was collected from the PL fractions, solvent was evaporated to dryness and the residue was redissolved in $300 \mu \mathrm{L}$ of methanol. Aliquots of $5 \mu \mathrm{L}$ were injected into a Gemini C18 HPLC column $(150 \times 2 \mathrm{~mm}$ ID, $3 \mu \mathrm{m}$ (Phenomenex, Torrance, CA, USA) thermostated at $35^{\circ} \mathrm{C}$. The mobile phase flow rate was $250 \mu \mathrm{L} \cdot \mathrm{min}-1$ with gradient elution of $\mathrm{A}: \mathrm{B}: \mathrm{C}(\mathrm{A}=10 \mathrm{mM}$ ammonium acetate in methanol with ammonia (0.025\%), $B=10 \mathrm{mM}$ ammonium acetate in water, $\mathrm{C}=$ isopropanol MeOH 8:2) - 0 min: 92:8:0, 7 min: 97:3:0, 12 min: 100:0:0, 19 min: 93:0:7, 20-23 min: 90:0:10, $24 \mathrm{~min}: 100: 0: 0$ and for equilibration of column 26-45 min: 92:8:0. The ESI/MS was carried out either in the positive or the negative ion detection mode at potential $+3 \mathrm{kV}$ or $-2.5 \mathrm{kV}$, with capillary temperature of $200{ }^{\circ} \mathrm{C}$. Eluting ions were detected with full scan mode from 200 to 1000 Da with the collisionally induced MS2 fragmentations (collision energy 35\%). Data were acquired and processed by means of XCalibur 2.1 software (Thermo Fisher Scientific). The responses of analyzed phospholipids were corrected by comparison to the signals of internal lipid standards that were obtained from Avanti Polar Lipids (Alabaster, AL, USA). As internal standards, we have used 17C FAs which are absent in our insect samples (PC_17:0/17:0 and PE_17:0/17:0 at $40 \mu \mathrm{g} / \mathrm{ml}$ and PG_17:0/17:0 and PS_17:0/17:0 at $20 \mu \mathrm{g} / \mathrm{ml})$. The corrected areas under individual analytical peaks were expressed in percentages assuming that the total area is $100 \%$.

\subsection{Statistics}

Survival data were analyzed using a generalized linear model with logistic link function for binary outcome. The survival data was dependent on stress duration ( 1 to 10 days), sex, temperature $\left(2,3,4,5^{\circ} \mathrm{C}\right)$ and thermal regime (CLT vs. FTR) as well as the second-order interactions. The two replicated experiments (2013 and 2014) were analyzed separately. Variations in the level of all individual metabolites or PLs were analyzed using analysis of variance (ANOVA) with thermal treatment (CLT, Fb, FA and CO) and stress duration (1, 2, 3 days) as crossed factors. To compare the temporal metabolic and lipidomic profiles among the different conditions, a between-class Principal Component Analysis (PCA) was used to identify the main patterns and clustering. A Monte Carlo test was then performed to examine the significance of the difference among the classes (based on 1000 simulations). To identify the variables (i.e. metabolites or PLs) contributing the most to the structure separation, the correlations to the principal components were extracted and ranked. Data were scaled and mean-centered prior to the PCAs. PLs composition and calculated indices were analyzed within each sampling duration using one-way ANOVA followed by Tukey's multiple comparison tests. Analyzes were performed using the statistical software ' $R$ 3.0.3'. 


\section{Results}

\subsection{Cold survival under constant and fluctuating regimes}

Mortality scored $4 \mathrm{~h}$ after exposing the flies to CLT or FTR is shown in Fig. 2. As expected, mortality was higher at lower temperatures and increased when stress duration increased in both sexes. However, the survival was clearly superior under FTR compared to CLT, particularly in females and at the highest tested temperature $\left(5^{\circ} \mathrm{C}\right)$, and this was observed in both experiment replicates. The statistical analyzes are summarized in Table 1 for both replications separately. All the main effects were significant, as well as most of the second order interactions. Nearly identical survival patterns were observed when mortality was scored after $24 \mathrm{~h}$ of recovery (presented in Supplemental Fig. S1).

As we found that benefits of FTRs were particularly manifested in females exposed to $5{ }^{\circ} \mathrm{C}$, this condition was used for temporal Omics profiling over the three first days (Fig. 1). No mortality was observed in any of these treatments, except after 3 days at $20^{\circ} \mathrm{C}$ where some females died (5\%) likely because of starvation effects which negatively interfered at this temperature. Therefore, we decided to exclude this specific condition ( 3 days at $20^{\circ} \mathrm{C}$ ) from all further analyzes.

\subsection{Metabolic profiling}

Among the 63 metabolites included in our spectral library, 43 were detected in the samples. The list of detected metabolites with their abbreviations is available in Table S1A and raw data for individual metabolites are provided in Table S1B. Among these 43 compounds, we found free amino acids (13), gamma amino acid (1), sugars (8), polyols (8), carboxylic acids (5), acidic sugar (glyceric acid), polyamines (3), alpha hydroxyl acid (1) and other molecules (3). Trehalose, glucose, proline and glutamine were the most abundant metabolites detected in whole-body extracts. Changes of all individual metabolite levels in relation to treatments and durations are illustrated in the panel Supplemental Fig. S2 and the corresponding univariate statistical outputs are provided in Table S2A. Changes of metabotype according to treatments were characterized using between-classes PCA and the ordination of classes within the first plane is presented in Fig. 3A. PC1 (53.8\%) and PC2 (20.9\%) cumulated $74.7 \%$ of total inertia (Fig. 3B). PC3 accounted for only $8 \%$ of total inertia and mainly represented within-treatment inertia. The Monte-Carlo randomizations confirmed the significance of the differences among classes $(P<0.001)$. The PC1 and PC2 scores (i.e. projection of centroids) of each treatment group are shown in Fig. $3 \mathrm{C}$ and $\mathrm{D}$. These analyzes revealed a clear opposition between all treatments exposed to cold (CLT1 to 3 and Fb1) that were negatively correlated to PC1 and the other treatments that were positively correlated to PC1. From Fig. 3C, it became clear that metabolic profiles departed significantly from their initial state (i.e. CO) in all CLT treatments, showing a strong homeostatic deviation, before coming back to control state during the fluctuations (especially after $2 \mathrm{~h}$ recovery). PC2 explained the time-course of the metabolic response from day 1 (positive association) to day3 (negative association), day2 being intermediate. The correlation of each metabolite to PC1 and PC2 are shown in Supplemental Fig. S3 A \& S3B. Among the metabolites contributing the most to PC1, we found almost all the detected polyols (arabitol, erythritol, sorbitol, mannitol, glycerol), some amino acids (Ser, Ala, Glu) and sugars (Man, Fru) that were negatively correlated to PC1 (i.e. more abundant in CLT flies) (see Supplemental Figs. S2 and S3 A). Some of these compounds showed rather large magnitude of fold-change accumulation compared to CO flies (e.g. erythritol: 22 fold, Man: 12 fold, Fru: 12 fold, sorbitol: 5 fold). Succinate, Tyr, inositol and Pro were among the few metabolites that were positively correlated to PC1, and thus more abundant in CO
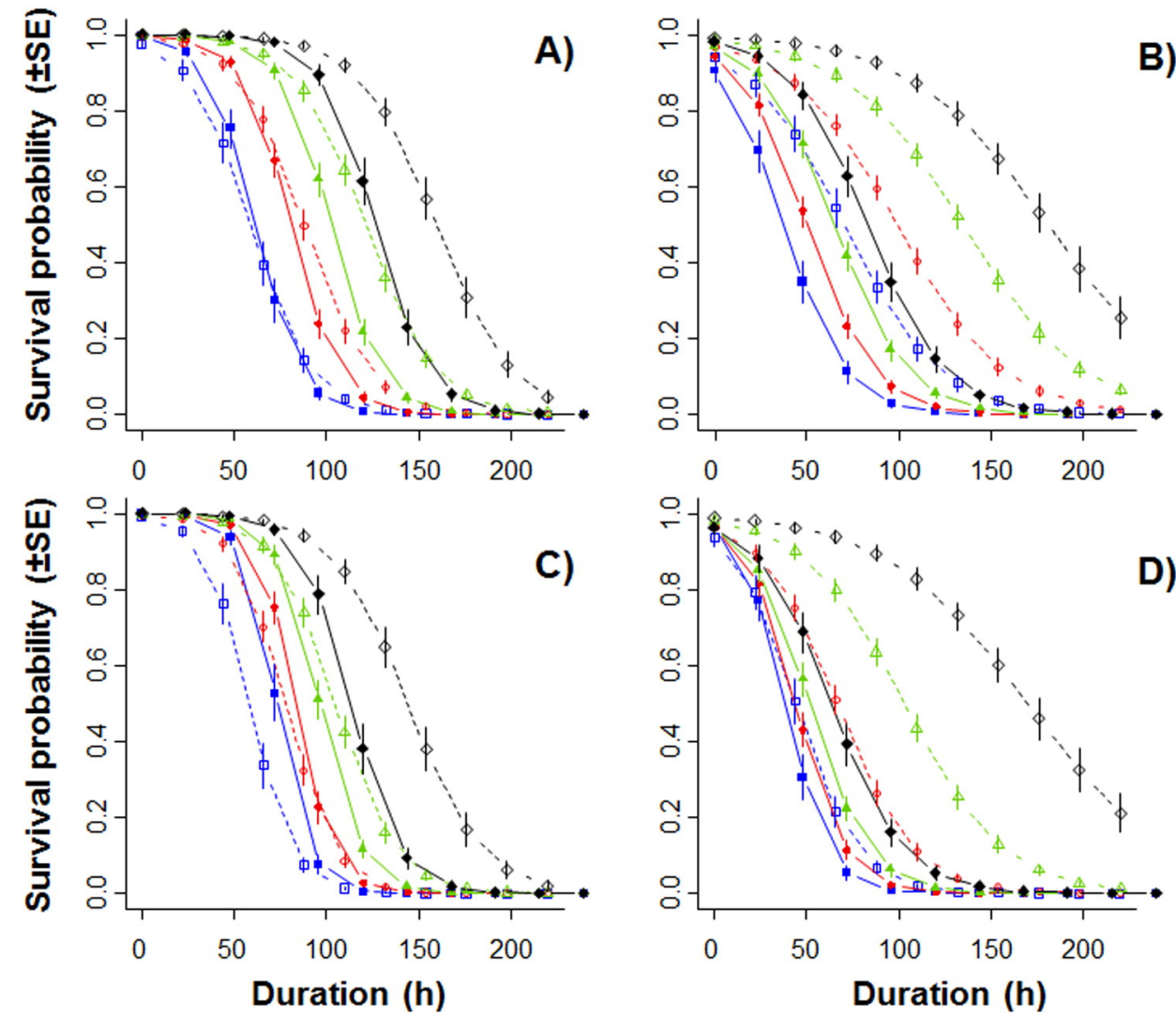

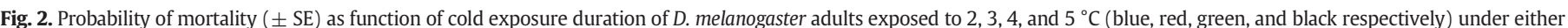

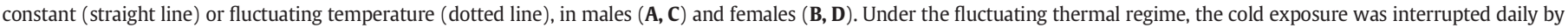

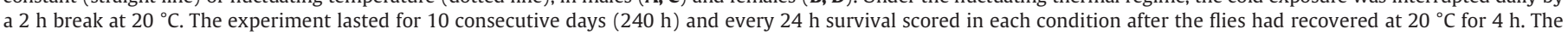

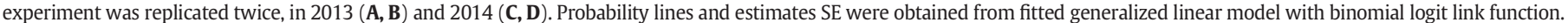


Table 1

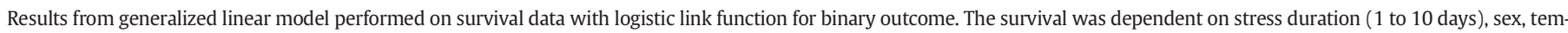
perature $\left(2,3,4,5{ }^{\circ} \mathrm{C}\right)$ and thermal regime (CLT vs. FTR). The two replicated experiments (2013 and 2014) were analyzed separately.

\begin{tabular}{|c|c|c|c|c|c|c|c|}
\hline Source (2013 experiment) & $C h i^{2}$ & $D f$ & $P$ & Source (2014 experiment) & $C h i^{2}$ & $D f$ & $P$ \\
\hline sex & 11.81 & 1 & 0.001 & sex & 60.18 & 1 & $<0.001$ \\
\hline temperature & 389.29 & 1 & $<0.001$ & temperature & 338.28 & 1 & $<0.001$ \\
\hline regime & 162.26 & 1 & $<0.001$ & regime & 85.67 & 1 & $<0.001$ \\
\hline duration & 1955.97 & 1 & $<0.001$ & duration & 2081.56 & 1 & $<0.001$ \\
\hline sex*regime & 34.57 & 1 & $<0.001$ & sex*regime & 44.7 & 1 & $<0.001$ \\
\hline regime*duration & 24.1 & 1 & $<0.001$ & regime*duration & 22.77 & 1 & $<0.001$ \\
\hline sex*duration & 29.24 & 1 & $<0.001$ & sex*duration & 26.19 & 1 & $<0.001$ \\
\hline sex*temperature & 17.04 & 1 & $<0.001$ & sex*temperature & 1.04 & 1 & 0.308 \\
\hline temperature*regime & 0.77 & 1 & 0.380 & temperature*regime & 11.54 & 1 & $<0.001$ \\
\hline temperature* duration & 7.4 & 1 & 0.007 & temperature* duration & 40.13 & 1 & $<0.001$ \\
\hline
\end{tabular}

and/or Fa flies (Supplemental Fig. S3 A). Concerning PC2, amino acids (Thr, Lys, Orn) and polyamines (putrescine and spermidine) were the most positively correlated metabolites (i.e. temporal increase over the 3 days of experiment), while other amino acids (Ile, Leu, Val) and sugars (Tre and Glc) were negatively correlated to PC2 (i.e. temporal decrease over time) (Supplemental Figs. S2 and S3B).

\subsection{Phospholipids profiling and patterns}

The LC/ESI/MS analysis detected 101 different PL molecular species belonging to different classes: phosphatidylethanolamine (PE), phosphatidylcholine (PC), phosphatidylglycerol (PG), phosphatidylinositol (PI), phosphatidylserine (PS), lyso-PE (LPE), lyso-PC (LPC), lyso-PG (LPG), lyso-PI (LPI), and monogalactosyl diglyceride (MGDG). Mean relative proportions of all phospholipids detected within all experimental conditions are summarized in Supplemental Table S3 (analytical data). Relative proportions of each PL class and fatty acyl, as well as calculated indices are provided in Supplemental Table S4 (summarized data). The dominant PLs classes in our whole-body extract samples were PCs (47-52\%) and PEs (37-41\%), followed by minor PIs (4-5\%), PGs (3-4\%), PSs (1-2\%) and Lyso-PLS (1\%). This pattern follows the general trend in insects [40] and fits with Drosophila lipidome [41]. Most of the PL molecular species exhibited statistically significant changes according to thermal treatment (48\%) and/or duration of exposure (37\%) (Supplemental Table S2B). The compounds PC_16:1/16:1 and PC_18:1/16:1 were the most abundant PLs; their combined relative proportion contributed up to $20 \%$ to the total PLs. The mean changes in the relative proportions of different PL classes according to treatment and durations are summarized in Fig. 4 and in Supplemental Table S4. Some changes in relative proportions among treatments were statistically significant in some PL classes (ANOVA, $P<0.05$ ) (Fig. 4). The most striking changes were: (i) a consistent increase in PEs in all the
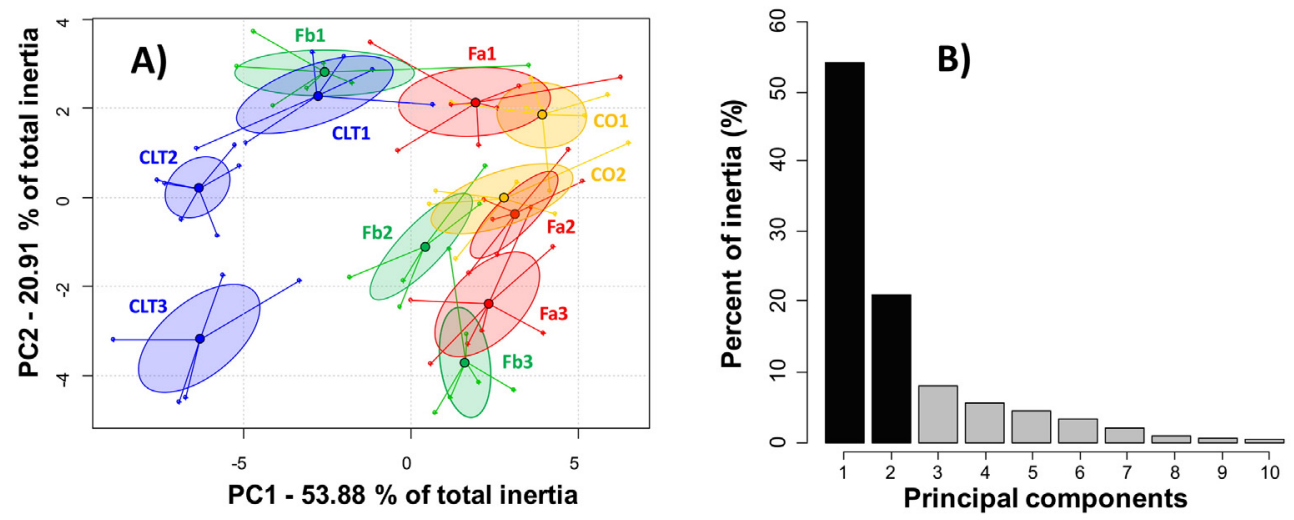

C)
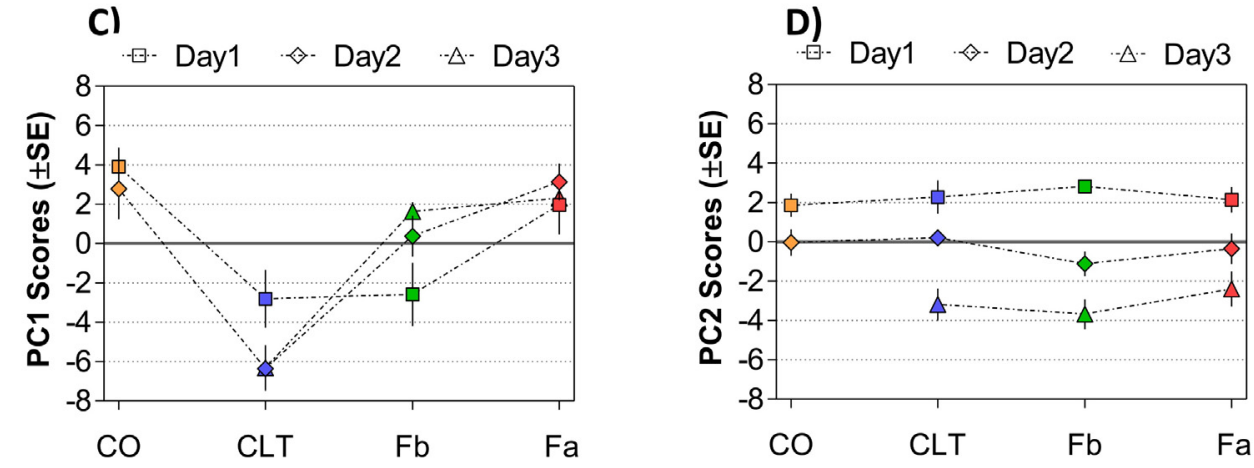

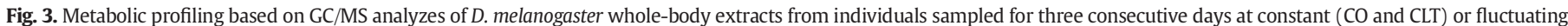

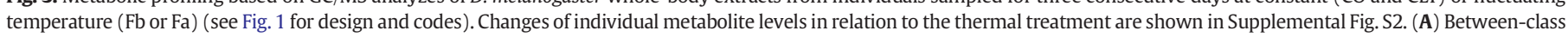

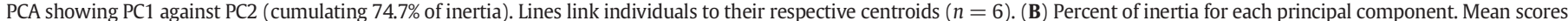

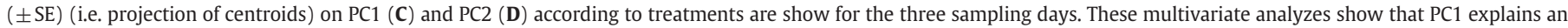

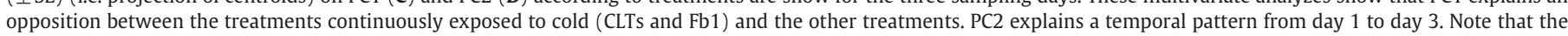
condition CO3 was discarded (see Section 3.1 for details). Correlations of the different metabolite concentrations to PC1 and PC2 are illustrated in Supplemental Fig. S3. 

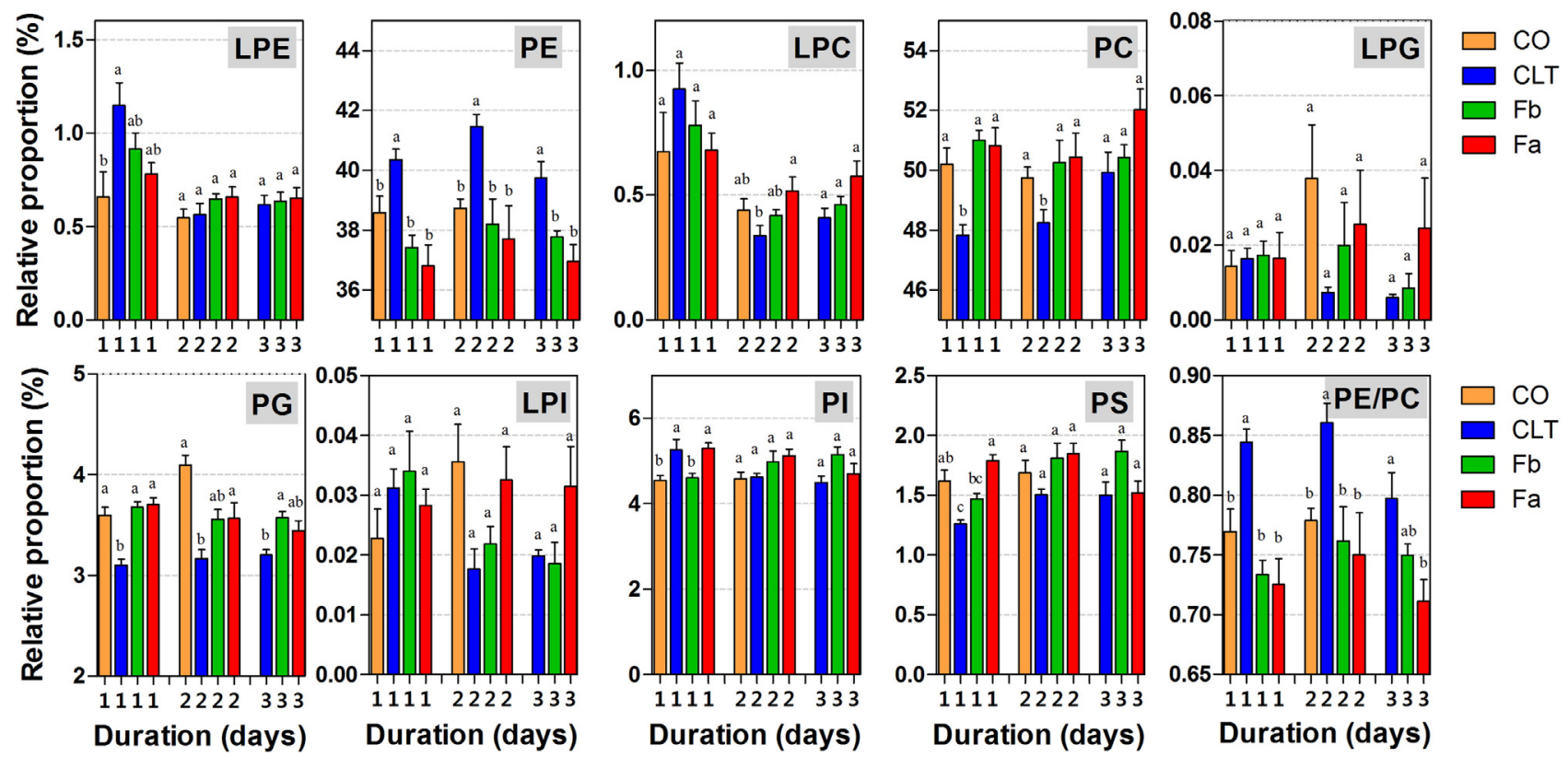

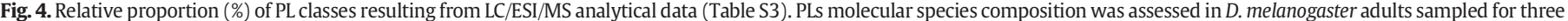

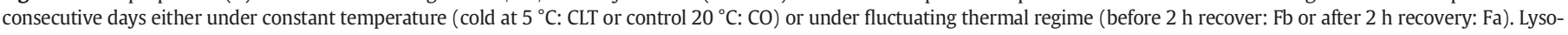

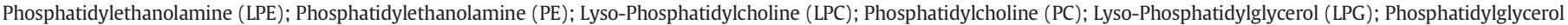

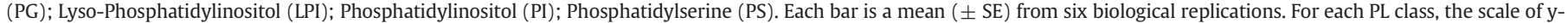

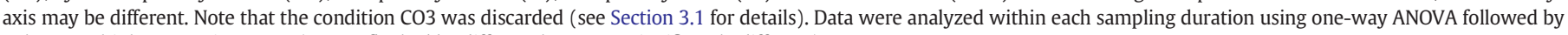
Tukey's multiple comparison tests (means flanked by different letters are significantly different).

CLT groups, and (ii) a corresponding decrease in PGs and PCs in the same treatments. It resulted that PE/PC ratio was strongly increased in individuals continuously exposed to cold (CLTs). The relative proportion LPEs also increased at cold (CLTs), but this was only manifested on the first day (Fig. 4).

Indices of PLs remodeling were calculated from LC/ESI/MS analytical data (Supplemental Table S3) and are summarized in Fig. 5 and in Supplemental Table S4. The most notable changes concerned the length of FA chains. Because 16C and 18C FAs prevail in membrane lipids of most insects, average chain length is often estimated based on the ratio of $16 \mathrm{C} / 18 \mathrm{C}$. The ratios short/long and the $16 \mathrm{C} / 18 \mathrm{C}$ both indicated that FA chains tended to be shorter in treatments exposed to low temperature (CLT and $\mathrm{Fb}$ ) compared to control at $20^{\circ} \mathrm{C}$ (CO) (ANOVA, $P<0.05$ ) (Fig. 5). After $2 \mathrm{~h}$ of recovery at $20{ }^{\circ} \mathrm{C}$ (i.e. treatments $\mathrm{Fa}$ ), the FA chains tended to be of intermediate length between cold and control, suggesting a fast return towards the initial condition during the $2 \mathrm{~h}$ recovery at $20{ }^{\circ} \mathrm{C}$. Concerning the indices of FAs desaturation (unsaturation index, UFA/SFA, PUFA/MUFA, Supplemental Table S4), we did not detect any consistent change according to treatments, except a small variation on the second day due to CO flies only (Fig. 5).

Global changes of lipidotype according to treatments clearly separated the groups into different clusters corresponding to the different treatments (Fig. 6A \& B). PC1 and PC2 accounted for 35.2 and $20.7 \%$ inertia. PC3 was also considered here as it still accounted for $15.9 \%$ of total inertia. Together, these three components cumulated $71.8 \%$ of the total inertia (Fig. 6C). The Monte-Carlo randomizations confirmed the significance of the differences among classes $(P<0.001)$. The projection of the scores on PC1, PC2 and PC3 are illustrated in Fig. 6D-F. Together, these multivariate analyzes revealed patterns that are strikingly consistent with those observed with the metabolic profiling. Explicitly, constant cold treatments (CLTs) were all negatively associated with PC1, while treatments exposed to $20^{\circ} \mathrm{C}$, either constantly (CO1 and $\mathrm{CO} 2$ ) or transiently (Fa1 to 3 ) were positively associated with PC1. This means that lipidomic profiles strongly deviated from their initial state in CLT flies before tending towards control state after $2 \mathrm{~h}$ recovery (Fig. 6A,D). PC2 was positively associated with a cluster that comprised
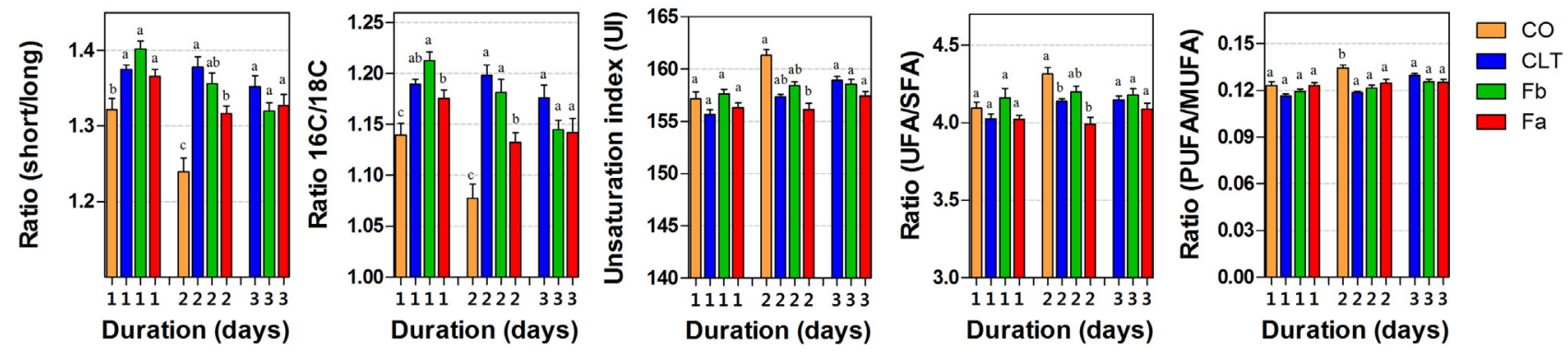

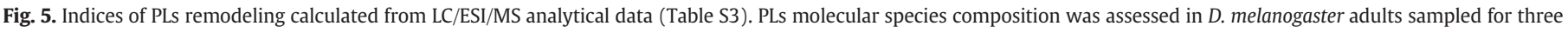

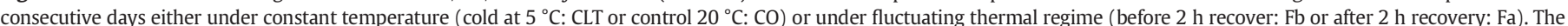

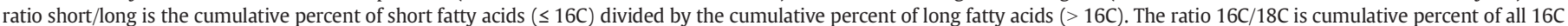

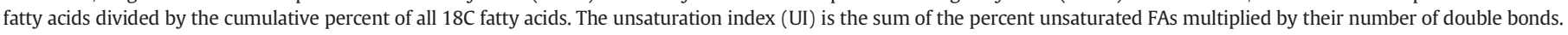

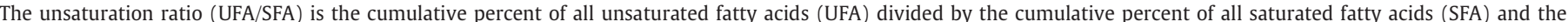

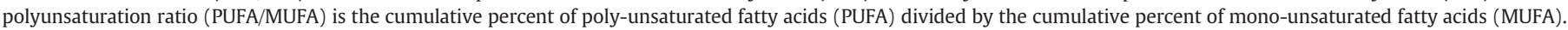

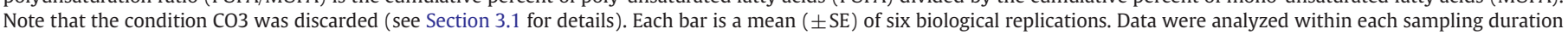
using one-way ANOVA followed by Tukey's multiple comparison tests (means flanked by different letters are significantly different). 


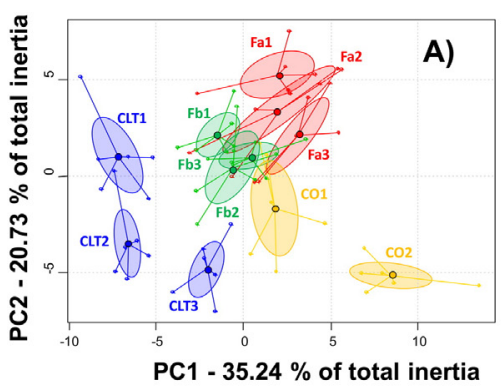

D)

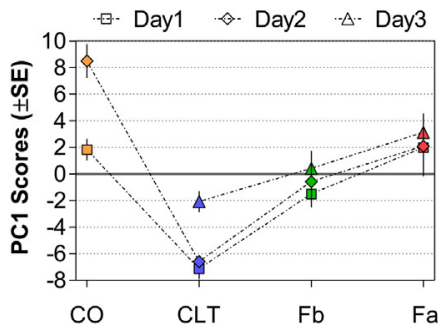

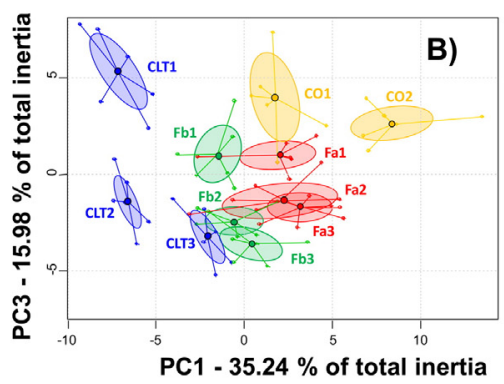

E)

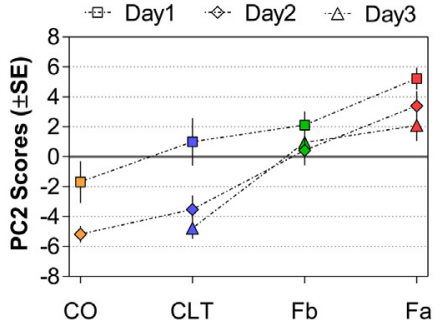

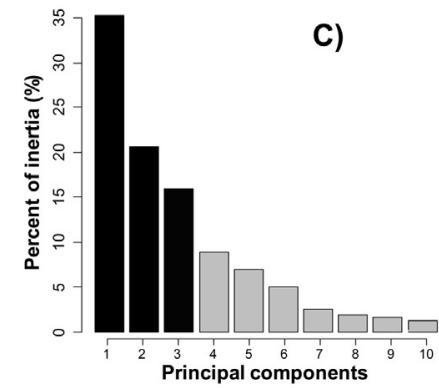

F)

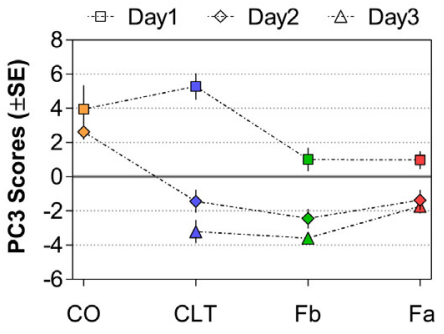

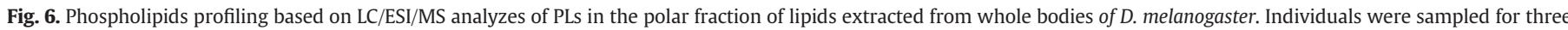

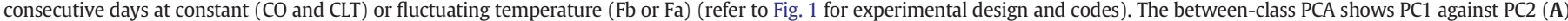

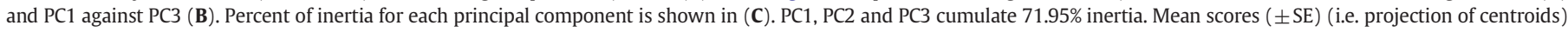

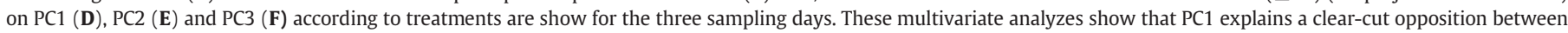

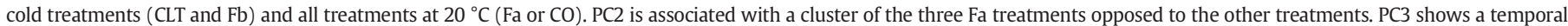

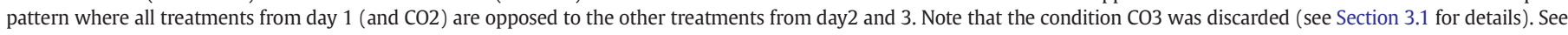
Supplemental Fig. S4(A,B,C) for correlations of PLs to the three PCA axes.

all fluctuating treatments (mostly Fa treatments) (Fig. 6A,E), and PC3 explained a temporal pattern where all treatments from day1 (as well as $\mathrm{CO} 2$ ) were opposed to the other treatments from day2 and 3 (Fig. 6B,F).

The variables (PLs) contributing the most to the structure separation along PC1, PC2 and PC3 were ranked according to their correlation value and are presented in Supplemental Fig. S4 A,B,C. From these, we found that short PEs (e.g. PE_16:1/14:0, PE_14:0/18:1, PE_16:1/16:0) and LPEs (e.g. LPE_16:0, LPE_14:0, LPE_18:0) were strongly negatively correlated to PC1 (i.e. relatively more abundant in CLTs), while PGs (e.g. PG_18:2/18:1, PG_18:3/18:1, PG_16:0/18:2) and PCs (PC_14:0/ 12:0, PC_18:2/18:1, PC_18:0/18:1) were strongly positively correlated to PC1 (i.e. relatively more abundant in COs and FAs) (Supplemental Fig. S4 A). The PLs that were the most positively correlated to PC2 included some PIs (e.g. PI_18:0/18:1, PI_16:0/16:1, PI_18:1/18:1) and PCs (e.g. PC_16:0/16:1, PC_16:0/14:0, PC_16:1/12:0). These were relatively more abundant in Fa flies, so after $2 \mathrm{~h}$ recovery (Supplemental Fig. S4B). Finally, the PLs that were the most positively correlated to PC3 (i.e. more abundant at the start of experiment on day1) comprised exclusively Lysp-PLs (e.g. LPG_16:1, LPC_14:0, LPC_16:1, LPE_18:1), while those that were negatively correlated (i.e. less abundant at the start of experiment) comprised various PLs such as PS_18/2:18:2, PC_16/0:16:0, and PE_14/1:12:0 (Supplemental Fig. S4C).

\section{Discussion}

In accordance with the literature on other insects, we confirmed that applying FTRs to D. melanogaster adults significantly reduces coldinduced mortality. Our findings provide additional support for the general occurrence of FTR-linked improvement of cold tolerance across insect taxa [2]. The promoting effect of FTR was particularly manifested in females and at the least stressful temperatures $\left(4\right.$ and $\left.5{ }^{\circ} \mathrm{C}\right)$. It is conceivable that at the lower temperatures ( 2 and $3{ }^{\circ} \mathrm{C}$ ), physiological damages had reached levels that cannot be fully counterbalanced within $2 \mathrm{~h}$ of daily recovery. We further assessed whether such a shift in cold tolerance phenotype is associated with detectable physiological changes. Repairing mechanisms under FTRs are under investigation but are still rather hypothetical. However, it appears clear that benefits of FTRs are somewhat related the re-establishment of overall ion homeostasis and electrochemical potentials across specific membranes during warm spells [30]. That is because chilling may induce separation of phospholipids bilayer and alter the permeability, and may also reduce the activity of membrane-bound enzymes, including the primary ionpumping systems such as $\mathrm{Na}^{+} / \mathrm{K}^{+}$-ATPase $[14,15,24]$. As a result, the ion homeostasis is disturbed at cold and the electrochemical membrane potentials partially or completely dissipate which leads to rapid development of chill injury and mortality $[17-20,24]$. The positive effect of warm periods under FTR is likely realized via supporting the active reestablishment of ion homeostasis by allowing the functionality of ATPgenerating pathways and ion pumping ATPases, as indicated in Pyrrhocoris apterus and Alphitobius diaperinus [30]. Theoretically, membrane phospholipid composition could be adaptively remodeled during warm periods in a direction that would counterbalance the negative effects of cold on membrane functions as mentioned above. Moreover, the cryoprotectants could mitigate the alterations in biological membranes by stabilizing their structures [26-29]. Consequently, we expected some changes in membrane phospholipid composition and/or in the concentrations of compounds with cryoprotective functions (polyols, sugars, amino acids) under FTRs. We did observe increasing levels in several polyhydric alcohols (arabitol, erythritol, sorbitol, mannitol, glycerol), sugars (Fru, Man) and amino acids (Ser, Ala, Glu). However, these changes were manifested in CLT treatments not in FTR treatments. Prolonged chilling may be required to trigger these accumulations and periodic warmings may offset this response. Glycerol and many other polyols (e.g ribitol, sorbitol and myo-inositol) have often been associated with cold-tolerance e.g. [23,27,42,43]. Similarly, prolonged exposure to moderately low temperature $\left(4^{\circ} \mathrm{C}\right)$ elevated levels of several polyols (glycerol, sorbitol) and amino acids (Ala, Glu) in Sarcophaga crassipalpis [44]. In beetle A. diaperinus exposed to cycling regimes ( $12 \mathrm{~h}$ cold $/ 12 \mathrm{~h}$ warm), changes in the concentration of cryoprotectants (glycerol and glucose) were observed only after the end of cold period and turned back to control value after the warm period [45]. The only metabolites that showed higher level at the end of the warm recovery were succinate, Tyr and Pro. Relative increased in Pro could be of interest as 
this free amino acid is a particularly potent cryoprotectant [46,47]. However, we consider it premature to speculate that Pro has causally contributed to cold tolerance of FTR flies as the concentrations and the magnitude of the fold-change accumulations remained low (1.5 fold). Because metabolite levels result from flux of many metabolic pathways and processes, it is difficult to depict whether the observed changes represent a beneficial (protective) or detrimental (dysregulation) signal; it may be a combination of both depending on the metabolite considered. In spite of this, metabolic profiling can be useful to monitor temporal changes of overall homeostasis under specific treatments [3234]. There is evidence for strong deviation of metabolic profiles when chill-susceptible insects are exposed to chilling [33,34,48,49]. It is not yet clear whether this marked deviation reflects only a degenerative syndrome, resulting for instance from uncoupling among various metabolic pathways at cold [44], or whether it partially entails a compensatory protective response, such as synthesis cryoprotectants. However, when insects are either adapted (genetically) or acclimated (plastically) to low temperature, they clearly possess the ability to hamper this cold-induced homeostatic deviation [33,34,49], suggesting that robustness in metabolic networks are key element of cold tolerance. No study so far has investigated the temporal maintenance/deviation of metabolic networks over the course of FTRs. We observed that metabolic trajectories deviated markedly from the control state during cold exposure, suggesting that flies under CLT were in a physiological state distinctively divergent and manifestly unfavorable compared to counterparts. Most importantly, we confirmed that warm intervals allowed a fast homeostatic regeneration towards initial state. Recovery as short as $2 \mathrm{~h}$ was sufficient to periodically return the metabolic system to functionality (as suggested by longer survival under FTR). We argue that periodic opportunities to restore metabolic networks contributes to cold tolerance of flies under FTRs.

Number of previous studies have investigated PLs composition of D. melanogaster in response to thermal acclimation [50-52] or thermal adaptation [53-56]; however, so far no study has analyzed temporal remodeling of PLs during rapid temperature shifts under FTRs. In addition, these earlier studies have generally analyzed the composition of FAs and/or head groups separately and in limited number of molecular species (often $<20$ species). Here we provide structural information of a large set of PLs (101 different molecular species), including the information on PLs head groups and FA chains. This large dataset coupled with time-series measurements allowed us to assess whether a rapid restructuring occur under FTRs in a direction that would support theory of homeoviscous adaptation $[14,25]$ and/or protection of membrane integrity at low temperatures [15]. Concerning head groups, we repeatedly found a relative increase in PEs in all the CLT treatments, and a corresponding decrease in PGs and PCs. The biosynthesis of PEs includes several pathways in which free ethanolamine (ETA) and the amino acid Ser are key precursors [57]. Interestingly, Ser was among the most influential metabolite associated with PC1 (Supplemental Fig. S3 A). Both Ser and ETA were more abundant in CLT flies (Supplemental Fig. S2) which may relate to the PE accumulation in CLT flies. The relative proportion of PEs (ratio PE/PC) was significantly higher in CLT flies than in the other groups. Ectotherms can compensate for temperature-induced changes in the viscosity of membrane lipids by increasing the proportion of PE head groups at the expense of PC head groups [14,15]. The ethanolamine moiety occupies a smaller area than the choline. PEs have a conical conformation, while PCs are more cylindrical. It results that PEs pack much less efficiently into the lipid bilayer [58]. The increased relative proportion of PEs at cold can be an attempt to compensate for the ordering effects of low temperatures and prevent unregulated transition of membrane bilayer into the non-functional gel phase. In insects, relative increase in PEs occurs during gradual cold acclimation $[59,60]$, but also over shorter periods, as observed in response to rapid cold hardening $(\mathrm{RCH})$ [61] but [54]. The relative proportion LPEs also increased at cold (i.e. CLTs), but this was only observed on the first day. Lyso-PLs were found in membranes in relatively low quantities (around 1\%) which is consistent with earlier reports [39]. Lyso-PLs have the shape of an inverted cone which disrupts tight packing into membranes and decreases the order, and hence increases the fluidity. Increased relative proportion of LPEs at cold is thus consistent with the physical properties of the prevailing thermal conditions. The specific role of minor membrane phospholipid classes in shaping thermal responses are still poorly known in insects and only start to be unraveled [39].

We also noted that the increase in relative proportion of PEs at cold occurred at the expense of some PGs. Phosphatidylglycerols are mostly cylindrical and bilayer-forming PLs [62]. The concurrent decrease of tubular-conformed PGs and increase in levels of conical-conformed PEs may also contribute to counteract the ordering effect at cold. Interestingly, members of PGs are considered as high melting point PLs and are associated with chilling susceptibility in plants [63]. Phosphatidylglycerols-derived lipids are mitochondrial cardiolipin (CL) precursors; therefore, PGs have a crucial role in cell physiology, including in stress response because CL synthesis is highly regulated and modulated under stress situations [62]. The level of PGs parallels the rate of oxygen consumption and oxidative metabolism [41]. Therefore, decreased relative level of PGs at cold could also result from reduced aerobic metabolism, which is consistent with the reduced level of succinate at cold (Supplemental Figs. S2, S3 A).

Some molecular species of PIs were specifically more abundant in recovering Fa flies (highly correlated to PC2 in Supplemental Fig. S4B). The synthesis of PIs takes place by the condensation of CDP-diacylglycerol with inositol $[63,64]$. PI synthase activity is tightly regulated by inositol level [63]. Interestingly, all polyhydric alcohols detected in our metabolic profiles were at higher level in CLT flies, with the exception of inositol which was more abundant in Fa flies (see Supplemental Figs. S2, S3 A) and may have served as precursor of PIs synthesis. Laboratory acclimation experiments have shown that PIs are much less dependent on environmental temperature than the other PLs, which suggests that PIs have different roles from those of PEs and PCs in membrane function and temperature adaptation [65]. Indeed, PIs and phosphorylated derivatives (phosphoinositides) play a central role in cell signaling and membrane trafficking in eukaryotes [63]. At this stage it is premature to suggest that these functions are implicated in the recovery mechanisms occurring during warm intervals but it may be an area to explore, especially since members of phosphoinositide signaling pathway are well-known to mediate stress responses in plants and yeasts $[64,66]$.

We did not detect any consistent changes related to FA desaturation according to treatments. This is consistent with earlier reports that failed to detect any correlation between FA unsaturation and cold exposure in drosophilids $[50,51,53,54,59]$. On the contrary, the ratios $16 \mathrm{C} / 18 \mathrm{C}$ indicated that FA chains tended to be shorter in flies sampled from the cold (i.e. CLT and $\mathrm{Fb}$ ) compared to flies exposed to continuous $20^{\circ} \mathrm{C}(\mathrm{CO})$. After $2 \mathrm{~h}$ of recovery under FTR, the FA chains length tended to be intermediate, suggesting a partial remodeling towards the initial condition during the warm intervals. Owing to the greater area of hydrophobic interactions, the PLs containing long-chain FAs have a higher melting temperature than short-chain FAs [67]. The modulation of FA chain length may be a strategy for mitigating membrane's ordering and increased viscosity at cold. FA shortening is commonly found in insects exposed to cold [16]. In some species, FA desaturation did not markedly vary with cold treatment but the $16 \mathrm{C} / 18 \mathrm{C}$ ratio increases $[68,69]$. In fact, FA shortening and FA unsaturation can be alternatively used to achieve a similar function [16]. Previous reports have shown that lipid composition is adjustable within very short periods, as little as a few hours for instance in insects exposed to $\mathrm{RCH}[50,61]$ or in intertidal molluscs that deal with ample daily thermal variations [70,71]. Our global lipidomic profiles show that rapid changes in PLs occur under FTRs. The lipidotypes, which integrate all detected PLs, significantly differed according to treatments: they strongly deviated from their initial state in all CLT treatments. Also, the global lipid composition was biased towards the lower temperature of the cycle $\left(5^{\circ} \mathrm{C}\right)$ before the 
recovery and towards the higher temperature of the cycle $\left(20^{\circ} \mathrm{C}\right)$ by the end of recurrent recovery, which means that a fast homeostatic regeneration occurrs during warm intervals. The variations in membrane lipid composition reported in this study, being manifest within only a $2 \mathrm{~h}$ period, are among the fastest recognized adjustments to environmental change.

It is remarkable that lipidomic trajectories completely mirrored metabolic trajectories. This implies that warm intervals involve a system-wide homeostatic regeneration process, of which membrane remodeling is likely just a facet. Relatively rapid changes (e.g. diurnal fluctuations) in lipid composition have also been reported in plants and fishes and these data suggest that alterations in lipid composition and membrane viscosity do not occur passively in response to changes in external temperature [72-74]. Membrane viscosity is not just a direct reflection of external temperature but is subject to active homeostatic regulation, even when normal mechanisms are impaired [75]. The patterns of change in membrane lipid composition noted here are in accordance with thermal compensation of membrane function. Rapid adjustments in the lipid composition under FTRs may reset the optimal functioning membranes and that of associated proteins (e.g. ion pumping system). We argue that this likely participates in mitigating the accumulation chilling damages. Cell membrane is a primary site of chilling injury [76] and persisting exposure to low temperature can result in leakage of ions and other solutes across cell membranes exposed to cold [17-20]. The complemented restoration of metabolic and PLs homeostasis under FTRs must definitively contribute to offset these deleterious cumulative effects at continuous low temperature.

\subsection{Conclusion}

In this study, we have shown that FTRs promoted cold tolerance of D. melanogaster flies. We could not correlate this phenotypic change with accumulation of cryoprotectants under FTRs. However, we found that prolonged exposure to low temperature was associated with a marked deviation of metabolic homeostasis and that recovery as short as $2 \mathrm{~h}$ was sufficient to periodically return the metabolic system to functionality. Lipidomics revealed that relative proportion of PEs increased and FAs chains tended to be shorter in flies at cold, likely to compensate for the ordering effect of low temperature. A striking observation was remarkable correspondence in the time-course of changes between metabolic network and PLs profiles, both suggesting a fast homeostatic regeneration during warm intervals. Therefore, we conclude that this rapid restoration process likely contributes to offset the accumulation of chilling injuries. Our multi-Omics approach revealed a set of metabolites and PLs putatively linked to recovery process under FTRs. These represent good candidates for further targeted studies. Finally, the adjustments of certain classes of PLs over temperature shifts were connected with corresponding changes in the levels of some metabolites known as biosynthetic precursors. Using combinations of techniques such as stable isotope tracer studies will permit to causatively associate these changes. Pharmacological inhibition of membrane restructuring [77] could also be useful approach to depict the precise role of membrane properties on cold survival under constant and fluctuating thermal regimes.

Supplementary data to this article can be found online at http://dx. doi.org/10.1016/j.bbalip.2016.08.008.

\section{Transparency document}

The Transparency document associated with this article can be found, in online version.

\section{Acknowledgment}

We are grateful to Charly Jehan for technical help on cold tolerance assessment and to the Coordinated Research Project "D41025" carried out under the sponsorship of the IAEA for constructive discussions on this topic. We are grateful to Czech Science Foundation (project no. 13-18509S) for lipidomic analysis.

\section{References}

[1] S.L. Chown, S.W. Nicolson, Insect physiological ecology: Mechanisms and patterns, Oxford University Press, Oxford, UK, 2004.

[2] H. Colinet, B.J. Sinclair, P. Vernon, D. Renault, Insects in Fluctuating Thermal Environments, Annu. Rev. Entomol. 60 (2015) 123-140.

[3] O. Nedved, D. Lavy, H.A. Verhoef, Modelling the time-temperature relationship in cold injury and effect of high-temperature interruptions on survival in a chillsensitive collembolan, Funct. Ecol. 12 (1998) 816-824.

[4] D. Renault, O. Nedved, F. Hervant, P. Vernon, The importance of fluctuating thermal regimes for repairing chill injuries in the tropical beetle Alphitobius diaperinus (Coleoptera : Tenebrionidae) during exposure to low temperature, Physiol. Entomol. 29 (2004) 139-145.

[5] H. Colinet, D. Renault, T. Hance, P. Vernon, The impact of fluctuating therma regimes on the survival of a cold-exposed parasitic wasp, Aphidius colemani, Physiol. Entomol. 31 (2006) 234-240.

[6] H. Colinet, T.T.A. Nguyen, C. Cloutier, D. Michaud, T. Hance, Proteomic profiling of a parasitic wasp exposed to constant and fluctuating cold exposure, Insect Biochem. Mol. Biol. 37 (2007) 1177-1188.

[7] H. Colinet, T. Hance, Male Reproductive Potential of Aphidius colemani (Hymenoptera: Aphidiinae) Exposed to Constant or Fluctuating Thermal Regimens, Environ. Entomol. 38 (2009) 242-249.

[8] C.P. Chen, D.L. Denlinger, Reduction of cold injury in flies using an intermittent pulse of high-temperature, Cryobiology 29 (1992) 138-143.

[9] V.H. Dollo, S.X.X. Yi, R.E. Lee, High temperature pulses decrease indirect chilling injury and elevate ATP levels in the flesh fly, Sarcophaga crassipalpis, Cryobiology $60(2010) 351-353$

[10] G.D. Yocum, J.P. Rinehart, W.P. Kemp, Duration and frequency of a high temperature pulse affect survival of emergence-ready Megachile rotundata (Hymenoptera: Megachilidae) during low-temperature incubation, J. Econ. Entomol. 105 (2012) 14-19.

[11] Z. Hanč, O. Nedvěd, Chill injury at alternating temperatures in Orchesella cincta (Collembola: Entomobryidae) and Pyrrhocoris apterus (Heteroptera: Pyrrhocoridae), Eur. J. Entomol. 96 (1999) 165-168.

[12] H. Colinet, L. Lalouette, D. Renault, A model for the time - temperature - mortality relationship in the chill-susceptible beetle, Alphitobius diaperinus, exposed to fluctuating thermal regimes, J. Therm. Biol. 36 (2011) 403-408.

[13] N.M. Teets, D.L. Denlinger, Physiological mechanisms of seasonal and rapid coldhardening in insects, Physiol. Entomol. 38 (2013) 105-116.

[14] A.R. Cossins, Temperature Adaptation of Biological Membranes, Portland Press, London, 1994.

[15] I.R. Hazel, R. Hazel, Thermal Adaptation in Biological Membranes: Is homeoviscous adaptation the explanation? Annu. Rev. Physiol. 57 (1995) 19-42.

[16] V. Koštál, Cell structural modifications in insects at low temperatures, in: D.L. Denlinger, R.E. Lee Jr. (Eds.), Low temperature biology of insects, Cambridge University Press, Cambridge, UK, 2010.

[17] V. Koštál, J. Vambera, J. Bastl, On the nature of pre-freeze mortality in insects: water balance, ion homeostasis and energy charge in the adults of Pyrrhocoris apterus, I. Exp. Biol. 207 (2004) 1509-1521.

[18] V. Koštál, M. Yanagimoto, J. Bastl, Chilling-injury and disturbance of ion homeostasis in the coxal muscle of the tropical cockroach (Nauphoeta cinerea), Comp. Biochem. Physiol. B Biochem. Mol. Biol. 143 (2006) 171-179.

[19] H.A. MacMillan, C.M. Williams, J.F. Staples, B.J. Sinclair, Reestablishment of ion homeostasis during chill-coma recovery in the cricket Gryllus pennsylvanicus, Proc. Natl. Acad. Sci. U. S. A. 109 (2012) 20750-20755.

[20] H.A. MacMillan, J.L. Andersen, S.A. Davies, J. Overgaard, The capacity to maintain ion and water homeostasis underlies interspecific variation in Drosophila cold tolerance, Sci. Rep. 5 (2015) 18607.

[21] G.D. Yocum, J. Zdarek, K.H. Joplin, R.E. Lee, D.C. Smith, K.D. Manter, D.L. Denlinger Alteration of the eclosion rhythm and eclosion behavior in the flesh fly, Sarcophaga crassipalpis, by low and high temperature stress, J. Insect Physiol. 40 (1994) 13-21.

[22] J.S. Hosler, J.E. Burns, H.E. Esch, Flight muscle resting potential and species-specific differences in chill-coma, J. Insect Physiol. 46 (2000) 621-627.

[23] L.R. E. Jr., A primer on insect cold-tolerance, in: D.L. Denlinger, R.E. Lee Jr. (Eds.), Low temperature biology of insects, Cambridge University Press, Cambridge, UK, 2010.

[24] H.A. MacMillan, B.J. Sinclair, Mechanisms underlying insect chill-coma, J. Insect Physiol. 57 (2011) 12-20.

[25] M. Sinensky, Homeoviscous adaptation-a homeostatic process that regulates the viscosity of membrane lipids in Escherichia coli, Proc. Natl. Acad. Sci. U. S. A. 71 (1974) 522-525.

[26] N.M. Tsvetkova, P.J. Quinn, Compatible solutes modulate membrane lipid phase behavior, in: A.R. Cossins (Ed.), Temperature Adaptation of Biological Membranes, Portland Press, London, 1994.

[27] V. Koštál, M. Slachta, P. Šimek, Cryoprotective role of polyols independent of the increase in supercooling capacity in diapausing adults of Pyrrhocoris apterus (Heteroptera: Insecta), Comp. Biochem. Physiol. B Biochem. Mol. Biol. 130 (2001) 365-374.

[28] A. Korsloot, C.A.M. Van Gestel, N.M. Van Straalen, Environmental Stress and Cellular Response in Arthropods, CRC Press, Boca Rota, FL, USA, 2004. 
[29] P.H. Yancey, Organic osmolytes as compatible, metabolic and counteracting cytoprotectants in high osmolarity and other stresses, I. Exp. Biol. 208 (2005) 2819-2830.

[30] V. Koštál, D. Renault, A. Mehrabianová, J. Bastl, Insect cold tolerance and repair of chill-injury at fluctuating thermal regimes: role of ion homeostasis, Comp. Biochem. Physiol. A Mol. Integr. Physiol. 147 (2007) 231-238.

[31] D. Kültz, Molecular and evolutionary basis of the cellular stress response, Annu. Rev. Physiol. 67 (2005) 225-257.

[32] A. Malmendal, J. Overgaard, J.G. Bundy, J.G. Sørensen, N.C. Nielsen, V. Loeschcke, M. Holmstrup, Metabolomic profiling of heat stress: hardening and recovery of homeostasis in Drosophila, Am. J. Phys. Regul. Integr. Comp. Phys. 291 (2006) R205-R212.

[33] H. Colinet, V. Larvor, M. Laparie, D. Renault, Exploring the plastic response to cold acclimation through metabolomics, Funct. Ecol. 26 (2012) 711-722.

[34] C.M. Williams, M. Watanabe, M.R. Guarracino, M.B. Ferraro, A.S. Edison, T.J. Morgan, A.F.B. Boroujerdi, D.A. Hahn, Cold adaptation shapes the robustness of metabolic networks in Drosophila melanogaster, Evolution 68 (2014) 3505-3523.

[35] H. Colinet, D. Renault, Metabolic effects of $\mathrm{CO}(2)$ anaesthesia in Drosophila melanogaster, Biol. Lett. 8 (2012) 1050-1054.

[36] H. Colinet, T. Chertemps, I. Boulogne, D. Siaussat, Age-related decline of abiotic stress tolerance in young Drosophila melanogaster adults, J. Gerontol. Ser. A Biol. Sci. Med. Sci. glv193 (2015), http://dx.doi.org/10.1093/gerona/glv193.

[37] H. Colinet, V. Larvor, R. Bical, D. Renault, Dietary sugars affect cold tolerance of Drosophila melanogaster, Metabolomics 9 (2012) 608-622.

[38] A. Tomcala, M. Tollarová, J. Overgaard, P. Simek, V. Koštál, Seasonal acquisition of chill tolerance and restructuring of membrane glycerophospholipids in an overwintering insect: triggering by low temperature, desiccation and diapause progression, J. Exp. Biol. 209 (2006) 4102-4114.

[39] V. Koštál, T. Urban, L. Rimnáčová, P. Berková, P. Šimek, Seasonal changes in minor membrane phospholipid classes, sterols and tocopherols in overwintering insect, Pyrrhocoris apterus, J. Insect Physiol. 59 (2013) 934-941.

[40] R.G.H. Downer, Lipid metabolism, in: G.A. Kerkut, L.I. Gilbert (Eds.), Comprehensive Insect Physiology, Biochemistry and Pharmacology, vol. 10, Pergamon Press, Oxford, UK., 1985

[41] M. Carvalho, J.L. Sampaio, W. Palm, M. Brankatschk, S. Eaton, A. Shevchenko, Effects of diet and development on the Drosophila lipidome, Mol. Syst. Biol. 8 (2012) 600

[42] J.M. Storey, K.B. Storey, in: K.B. Storey (Ed.), Cold Hardiness and Freeze Tolerance, in: Functional Metabolism: Regulation and Adaptation, John Wiley \& Sons, Inc., Hoboken, NJ, USA, 2004.

[43] L. Vesala, T.S. Salminen, V. Koštál, H. Zahradnickova, A. Hoikkala, Myo-inositol as a main metabolite in overwintering flies: seasonal metabolomic profiles and cold stress tolerance in a northern drosophilid fly, J. Exp. Biol. 215 (2012) 2891-2897.

[44] M.R. Michaud, D.L. Denlinger, Shifts in the carbohydrate, polyol, and amino aci pools during rapid cold-hardening and diapause-associated cold-hardening in flesh flies (Sarcophaga crassipalpis): a metabolomic comparison, J. Comp. Physiol. B. 177 (2007) 753-763.

[45] L. Lalouette, V. Koštál, H. Colinet, D. Gagneul, D. Renault, Cold exposure and associated metabolic changes in adult tropical beetles exposed to fluctuating thermal regimes, FEBS J. 274 (2007) 1759-1767.

[46] V. Koštál, H. Zahradníčková, P. Šimek, Hyperprolinemic larvae of the drosophilid fly, Chymomyza costata, survive cryopreservation in liquid nitrogen, Proc. Natl. Acad. Sci. U. S. A. 108 (2011) 13041-13046.

[47] V. Koštál, P. Šimek, H. Zahradníčková, J. Cimlová, T. Štětina, Conversion of the chil susceptible fruit fly larva (Drosophila melanogaster) to a freeze tolerant organism, Proc. Natl. Acad. Sci. U. S. A. 109 (2012) 3270-3274.

[48] J. Overgaard, A. Malmendal, J.G. Sørensen, J.G. Bundy, V. Loeschcke, N.C. Nielsen, M. Homstrup, Metabolomic profiling of rapid cold hardening and cold shock in Drosophila melanogaster. J. Insect Physiol. 53 (2007) 1218-1232.

[49] N.M. Teets, J.T. Peyton, G.J. Ragland, H. Colinet, D. Renault, D.A. Hahn, D.L. Denlinger, Combined transcriptomic and metabolomic approach uncovers molecular mechanisms of cold tolerance in a temperate flesh fly, Physiol. Genomics 44 (2012) 764-777.

[50] J. Overgaard, J.G. Sørensen, S.O. Petersen, V. Loeschcke, M. Holmstrup, Changes in membrane lipid composition following rapid cold hardening in Drosophila melanogaster, J. Insect Physiol. 51 (2005) 1173-1182.

[51] J. Overgaard, J.G. Sørensen, S.O. Petersen, V. Loeschcke, M. Holmstrup, Reorganization of membrane lipids during fast and slow cold hardening in Drosophila melanogaster, Physiol. Entomol. 31 (2006) 328-335.

[52] V. Koštál, J. Korbelová, J. Rozsypal, H. Zahradníčková, J. CimLova, A. Tomčala, P. Šimek, Long-term cold acclimation extends survival time at $0{ }^{\circ} \mathrm{C}$ and modifies the metabolomic profiles of the larvae of the fruit fly Drosophila melanogaster, PLoS One 6 (2011) 20-25.
[53] T. Ohtsu, M.T. Kimura, C. Katagiri, How Drosophila species acquire cold tolerancequalitative changes of phospholipids, Eur. J. Biochem. 252 (1998) 608-611.

[54] H.A. MacMillan, C.G. Guglielmo, B.J. Sinclair, Membrane remodeling and glucose in Drosophila melanogaster: a test of rapid cold-hardening and chilling tolerance hypotheses, J. Insect Physiol. 55 (2009) 243-249.

[55] S.G. Goto, H. Udaka, C. Ueda, C. Katagiri, Fatty acids of membrane phospholipids in Drosophila melanogaster lines showing rapid and slow recovery from chill coma, Biochem. Biophys. Res. Commun. 391 (2010) 1251-1254.

[56] B.S. Cooper, L.A. Hammad, N.P. Fisher, J.A. Karty, K.L. Montooth, In a variable thermal environment selection favors greater plasticity of cell membranes in Drosophila melanogaster, Evolution 66 (2012) 1976-1984.

[57] G.B. Ansell, S. Spanner, Phosphatidylserine, phosphatidylethanolamine and phosphatidylcholine, in: J.N. Hawthorne, G.B. Ansell (Eds.), Phospholipids, Elsevier Biomedical Press, Amsterdam, 1982.

[58] J.R. Hazel, Cold adaptation in ectotherms: Regulation of membrane function and cellular metabolism, Advances in Comparative and Environmental Physiology, 4, 1989 1-50.

[59] J. Overgaard, A. Tomcala, J.G. Sørensen, M. Holmstrup, P.H. Krogh, P. Šimek, V. Koštál, Effect of acclimation temperature on thermal tolerance and membrane phospholipid composition in the fruit fly Drosophila melanogaster, J. Insect Physiol. 54 (2008) 619-629.

[60] S.G. Goto, C. Katagiri, Effects of acclimation temperature on membrane phospholipids in the flesh fly Sarcophaga similis, Entomol. Sci. 14 (2011) 224-229.

[61] M.R. Michaud, D.L. Denlinger, Oleic acid is elevated in cell membranes during rapid cold-hardening and pupal diapause in the flesh fly, Sarcophaga crassipalpis, J. Insect Physiol. 52 (2006) 1073-1082.

[62] L.A. Luévano-Martínez, A.J. Kowaltowski, Phosphatidylglycerol-derived phospholipids have a universal, domain-crossing role in stress responses, Arch. Biochem. Biophys. 585 (2015) 90-97.

[63] M.E. Gardocki, N. Jani, J.M. Lopes, Phosphatidylinositol biosynthesis: biochemistry and regulation, Biochim. Biophys. Acta 1735 (2005) 89-100.

[64] S.A. Henry, M.L. Gaspar, S.A. Jesch, The response to inositol: Regulation of glycerolipid metabolism and stress response signaling in yeast, Chem. Phys. Lipids 180 (2014) 23-43.

[65] R. Käkelä, M. Mattila, M. Hermansson, P. Haimi, A. Uphoff, V. Paajanen, P. Somerharju, M. Vornanen, Seasonal acclimatization of brain lipidome in a eurythermal fish (Carassius carassius) is mainly determined by temperature, Am. J. Phys. Regul. Integr. Comp. Phys. 294 (2008) R1716-R1728.

[66] H.W. Xue, X. Chen, Y. Mei, Function and regulation of phospholipid signalling in plants, Biochem. J. 421 (2009) 145-156.

[67] C. Huang, H. Lin, S. Li, G. Wang, Influence of the positions of cis double bonds in the sn-2-acyl chain of phosphatidylethanolamine on the bilayer's melting behavior, J. Biol. Chem. 272 (1997) 21917-21926.

[68] T. Ohtsu, C. Katagiri, M.T. Kimura, Biochemical Aspects of Climatic Adaptations in Drosophila curviceps, D. immigrans, and D. albomicans (Diptera: Drosophilidae), Environ. Entomol. 28 (1999) 968-972.

[69] M. Bashan, H. Akbas, K. Yurdakoc, Phospholipid and triacylglycerol fatty acid composition of major life stages of sunn pest, Eurygaster integriceps (Heteroptera: Scutelleridae), Comp. Biochem. Physiol. B Biochem. Mol. Biol. 132 (2002) 375-380.

[70] E. Williams, G. Somero, Seasonal-, tidal-cycle- and microhabitat-related variation in membrane order of phospholipid vesicles from gills of the intertidal mussel Mytilus californianus, J. Exp. Biol. 199 (1996) 1587-1596.

[71] F. Pernet, S. Gauthier-Clerc, É. Mayrand, Change in lipid composition in eastern oyster (Crassostrea virginica Gmelin) exposed to constant or fluctuating temperature regimes, Comp. Biochem. Physiol. B Biochem. Mol. Biol. 147 (2007) 557-565.

[72] J.R. Hazel, S.R. Landrey, Time course of thermal adaptation in plasma membranes of trout kidney. I. Headgroup composition, Am. J. Phys. 255 (1988) R622-R627.

[73] B.Y.C. Carey, J.R. Hazel, Diurnal Variation in Membrane Lipid Composition of Sonoran Desert Teleosts, J. Exp. Biol. 147 (1989) 375-391.

[74] A. Martinière, M. Shvedunova, A.J.W. Thomson, N.H. Evans, S. Penfield, J. Runions, H.G. Mcwatters, Homeostasis of plasma membrane viscosity in fluctuating temperatures, New Phytol. 192 (2011) 328-337.

[75] I. Guschina, J.L. Harwood, Mechanisms of temperature adaptation in poikilotherms, FEBS Lett. 580 (2006) 5477-5483.

[76] R.E. Lee Jr., K. Damodaran, S.X.X. Yi, G.A. Lorigan, Rapid cold-hardening increases membrane fluidity and cold tolerance of insect cells, Cryobiology 52 (2006) 459-463.

[77] G. Balogh, M. Péter, G. Liebisch, I. Horváth, Z. Török, E. Nagy, A. Maslyanko, S. Benko, G. Schmitz, J. Harwood, L. Vígh, Lipidomics reveals membrane lipid remodelling and release of potential lipid mediators during early stress responses in a murine melanoma cell line, Biochim. Biophys. Acta 1801 (2010) 1036-1047. 